\title{
DISEÑO DE UN CONTROLADOR DE INTENSIDAD LUMINOSA PARA UNA MÁQUINA DE INSOLACIÓN
}

\section{Design of controller for a machine light intensity insolation}

\author{
Javier Eduardo Martínez Baquero ${ }^{1}$, Elías Parrado S. ${ }^{2}$, Luis E. Morales Suarez ${ }^{3}$ \\ ${ }^{1}$ Universidad de los Llanos - Unillanos, Escuela de Ingeniería, Grupo Investigación GITECX, Colombia. \\ Email:jmartinez@unillanos.edu.co \\ ${ }^{2}$ Universidad de los Llanos-Unillanos, Especialista en Instrumentación y Control Industrial, Colombia. \\ Email: eliasparradosandoval@yahoo.com.mx \\ ${ }^{3}$ Universidad de los Llanos-Unillanos Especialista en Instrumentación y Control Industrial, Colombia. \\ Email: enriquemorales.s@hotmail.com
}

(Recibido Agosto 22 de 2016 y aceptado Octubre 21 de 2016)

\begin{abstract}
Resumen
El presente documento muestra el diseño de un controlador para una máquina de insolación usada en procesos de fotocurado. Para ello se inició el desarrollo realizando una revisión previa minuciosa a la máquina de insolación existente, y a los procedimientos que se deben llevar a cabo en el laboratorio de estampados, con el fin de comprender el proceso de fotocurado, evaluando las posibilidades de mejora de los equipos en uso. El diseño propuesto para el controlador es un $\mathrm{PI}$, el cual se realizó con herramientas de simulación de Matlab, dando prioridad al tiempo de exposición en el proceso de fotocurado el cual debe ser el mismo para cualquier tipo de tela o malla del bastidor, lo cual permite que la máquina de insolación no requiera ser operada por personal altamente calificado o competente en este arte.
\end{abstract}

Palabras clave: Serigrafía, insolación, control PI, diseño

\begin{abstract}
This paper presents a design for a insolation machine controller used in curing processes. This development began conducting a thorough prior review to the actual insolation machine and the procedures to be carried out in the printing laboratory, in order to understand the process of curing, assessing the possibilities for improving the equipment in use. The proposed controller design is a PID, which was made with matlab simulation tools, giving priority to the exposure time in the process of curing which must be the same for any type of fabric or mesh frame. This will allow the insolation machine does not require to be operated by highly qualified and competent staff in this art.
\end{abstract}

Key words: Serigraphy, insolation, PI control, design.

\section{INTRODUCCIÓN}

En aquellas empresas donde se realiza el proceso de fabricación de negativos de bastidores que van a ser utilizados en procesos de estampados, el éxito de esta labor depende en gran medida de la experiencia del operador del laboratorio, ya que este proceso al ser tan artesanal requiere de un conocimiento de las emulsiones y del uso de las máquinas de insolación, la cuales al ser de fabricación "casera" presentan demasiadas interferencias al sistema, siendo estos los factores determinantes para que la calidad de los estampados varíe en su calidad.

Este proceso de estampado, llamado serigrafía, del griego Serikós "seda" y Graphé "escribir", es una técnica de impresión que consiste en copiar imágenes, dibujos o texto sobre cualquier material, transfiriendo una tinta a través de una malla de seda, metal o nailon tensado en un marco, el paso de la tinta se bloquea en las áreas donde no habrá imagen mediante una emulsión o barniz, quedando libre la zona donde pasará la tinta. Duran-

Cómo citar este artículo:

Cómo citar este artículo: J.E. Martínez Baquero, E. Parrado \& L.E. Morales-Suarez, "Diseño de un controlador de intensidad luminosa para una máquina de insolación, “ Rev. Ingeniería Investigación y Desarrollo, vol. 17 N 1, pp. 26-36, Enero, 2017. 
te esta actividad, el proceso de insolación consiste en emulsionar con una resina fotosensible los bastidores, que son las pantallas enteladas con marcos en madera donde quedara grabado el diseño y servirá como elemento principal de plantilla para estampar, la emulsión fotosensible es sometida a la exposición de una potente fuente luminosa, cuya luz atraviesa los espacios que ha dejado al descubierto el diseño o positivo endureciendo la sustancia fotosensible.

Debido a lo anterior, se presenta a continuación el diseño del controlador de la intensidad luminosa para una máquina de insolación, desarrollada por estudiantes y docentes de la Especialización en Instrumentación y Control de la Universidad de los Llanos, con el cual se desean obtener mejores resultados en los procesos de estampados.

De esta manera, con el presente proyecto se desea incursionar aún más en el campo de la investigación, para aumentar la competitividad y así mejorar lo dicho por [1], quien argumenta que en general, la falta de competitividad en los sectores productivos de los países en vías de desarrollo, según los expertos en las sociedades latinoamericanas, tiene raíces profundas en la carencia de una cultura de la investigación, que se explica por la debilidad en este ámbito del sistema educativo y por el desconocimiento de su historia por la sociedad en esas naciones.

\section{FORMULACIÓN DEL PROBLEMA}

La intensidad de la luz juega un papel muy importante a la hora de realizar el proceso de fotocurado del bastidor en una máquina de insolación, definiendo la dureza de la emulsión diazoica en las zonas donde se desea que al lavar el bastidor se caiga la emulsión resultando el dibujo del diseño. Cuando se realiza un diseño de policromía se necesitan varios bastidores para formar la imagen del estampado, si el bastidor en el proceso de fotocurado falla en la definición del diseño en la emulsión, al sobreponer las imágenes resulta una figura borrosa.

Este problema se presenta con frecuencia, y conlleva a pérdidas en la producción debido al desperdicio de materia prima, principalmente porque el tiempo de exposición a la luz no es suficiente o no tiene la potencia adecuada que permita un resultado acorde con el diseño propuesto para el estampado.
En la figura 1 se muestra un error en la definición del proceso de fotocurado.

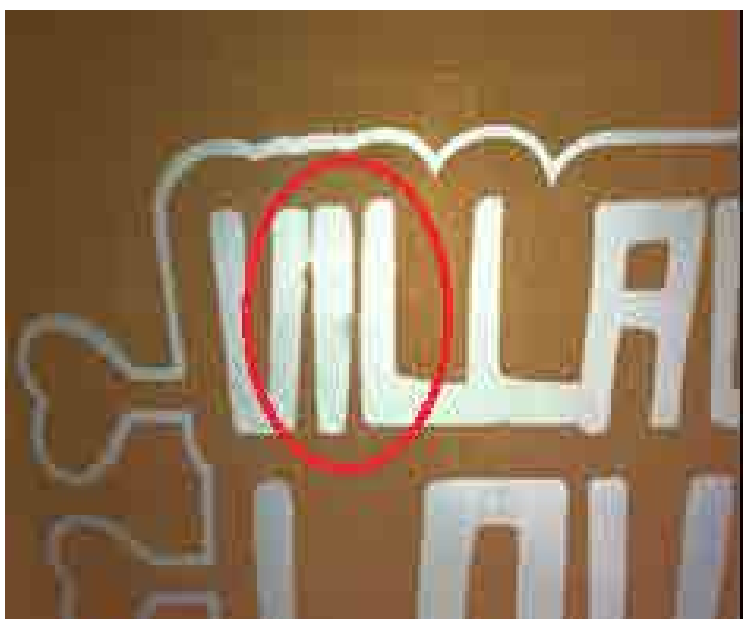

Figura 1. Error en definición proceso defotocurado

\section{MATERIALES Y MÉTODOS}

La automatización en la industria, hasta los años 70 , se ejecutaba exclusivamente con relés, conocida como una lógica cableada, pero a partir de estos años, la sistemas de automatización (Controladores Lógico Programables - PLC) interrumpieron ese camino y se trasladó a la lógica programada. Las primeras versiones y modelos de PLC eran costosos, difíciles de programar, tenían relativamente poca memoria y casi ningún periférico.

Las aplicaciones de automatización modernas deben ser capaces de adaptarse a los cambios en los requerimientos de producción. Dado que la mayoría de las aplicaciones de fabricación son distribuidas, puede ser necesario reconfigurar las operaciones que ejecutan varios dispositivos del sistema de forma sincronizada. Desafortunadamente, reconfigurar este tipo de aplicaciones es una tarea compleja para los desarrolladores de aplicaciones, especialmente cuando las operaciones se deben ejecutar en determinados instantes de tiempo o los recursos son escasos. [2].

Un sistema automatizado está conformado por elementos o instrumentos, lo cuales son utilizados para medir variables físicas, ejercer acciones de control y transmitir señales. En todos los procesos es absolutamente necesario controlar y mantener constantes algunas magnitudes. [3]. 
El diccionario de la Real Academia Española define la Automática como la disciplina que trata de los métodos y procedimientos cuya finalidad es la sustitución del operador humano por un operador artificial en la ejecución de una tarea física o mental previamente programada. [4].

Los sistemas de control realizan estas operaciones de forma automática, estabilizando la variable del proceso (salida) en un valor deseado (set point). Gracias al uso de los controladores se han mejorado muchos procesos de producción, incrementando la calidad de productos en la industria.

En la ecuación (1) se representa un controlador PID

$$
P I D=K p\left(1+\frac{1}{T i S}+T d S\right)
$$

Este controlador establece su característica de desempeño según la ecuación (2), como la señal en el dominio de tiempo llamada señal de control o señal de mando, indicando allí las constantes que lo rigen.

$$
\mathrm{u}(\mathrm{t})=\operatorname{Kpe}(\mathrm{t})+\frac{K p}{T i} \int \mathrm{e}(\mathrm{t}) d t+K p T d \frac{d}{d t} e(t)(2)
$$

Para la máquina de insolación se ha optado por un controlador $\mathrm{PI}$, considerando que la acción derivativa no se requiere debido a que el sobrepaso no será significativo. La ecuación (3) presenta el controlador PI.

$$
P I=K p\left(1+\frac{1}{T i S}\right)(3)
$$

Una vez identificado el controlador es importante mencionar que existen diversos métodos de diseño, entre ellos las reglas de Sintonización Ziegler Nichols, la cual se basa en la respuesta del sistema en lazo abierto ante una señal escalón. [5].

Para este proyecto se hace uso del primer método de Ziegler Nichols ya que la planta no presenta integradores. Para el cálculo de las constantes se toma como base la tabla 1.
Tabla 1. Sintonización de Controladores PID

\begin{tabular}{cccc}
\hline Tipo & $\mathbf{K p}$ & $\mathbf{T i}$ & $\mathbf{T d}$ \\
\hline $\mathbf{P}$ & $\mathrm{T} / \mathrm{L}$ & $\mathrm{INF}$ & 0 \\
$\mathbf{P I}$ & $0.9(\mathrm{~T} / \mathrm{L})$ & $\mathrm{L} / 0.3$ & 0 \\
$\mathbf{P I D}$ & $1.2(\mathrm{~T} / \mathrm{L})$ & $2 \mathrm{~L}$ & $0.5 \mathrm{~L}$
\end{tabular}

Fuente: Tomado del libro Ingeniería de Control Moderna.

El sistema de control de intensidad luminosa para la máquina de insolación estará determinado por el diagrama de bloques mostrado en la figura 2 , donde se establecen los elementos que allí intervienen.

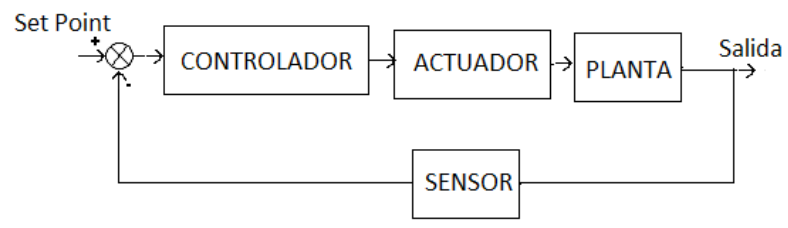

Figura 2. Diagrama de bloques sistema de control.

El empleo de los sensores en la tecnología, tanto en el ámbito industrial como doméstico, se ha vuelto usual la medición de magnitudes mecánicas, térmicas, eléctricas y químicas en sectores como industrias automatizadas, la robótica, la ingeniería experimental, el ahorro energético, el control ambiental, automóviles, electrodomésticos, computadoras, son tareas que serían impensables sin la aplicación de los sensores. [6].

Una vez establecido el tipo de controlador que será empleado para el sistema de control, se procede a su diseño, indicando que fue necesario la implementación de un prototipo de máquina de insolación diseñado con las dimensiones requeridas para los bastidores ya existentes. Los materiales utilizados para el prototipo fue madera, haciéndose un embudo en lámina galvanizada y ubicando un reflector en su parte inferior del embudo acondicionándose con papel de aluminio adhesivo para mejorar la proyección y dirección del haz de luz.

Con respecto al sensor de luz se hará uso de celdas fotovoltaicas por su eficiencia y facilidad de instalación, decidiendo la implementación de tecnología LED (Figura 3 ), además buscando bajo consumo de energía y reducir costos en la lámpara. 


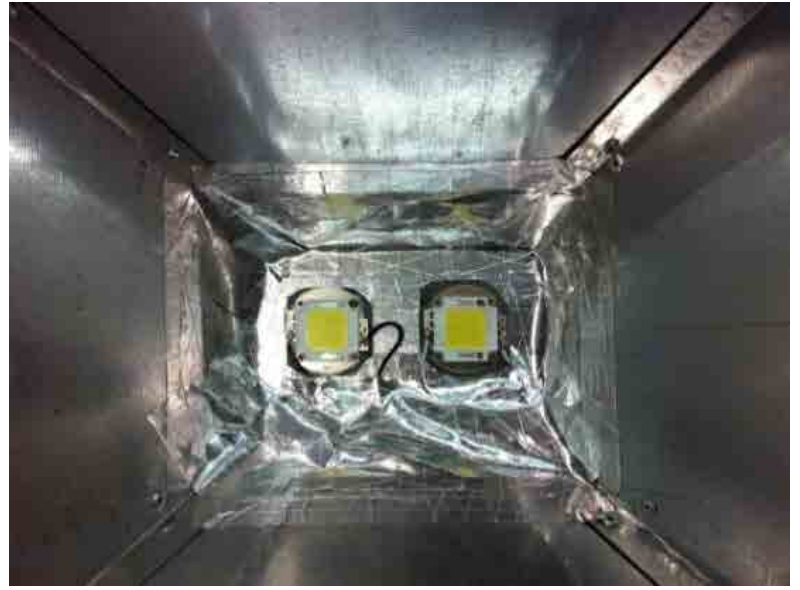

Figura 3. Lámparas LED instaladas en prototipo

Dichas celdas fotovoltaicas generan un voltaje DC en sus terminales proporcional a la luz incidente.

Posteriormente, con el fin de definir la variación del valor del voltaje de la celda fotovoltaica a la exposición de la luz en el área de influencia del haz, se procede a realizar mediciones para observar estos cambios. Para ello se dibujó una cuadricula de nueve por siete secciones en el vidrio de la máquina de insolación fabricada y se tomaron los datos registrados a continuación en la tabla 2.

Tabla 2. Voltajes obtenidos por el sensor en cada sección del vidrio.

\begin{tabular}{|l|l|l|l|l|l|l|l|l|}
\hline 4,488 & 4,518 & 4,555 & 4,595 & 4,637 & 4,620 & 4,542 & 4,548 & 4,477 \\
\hline 4,470 & 4,550 & 4,589 & 4,635 & 4,682 & 4,654 & 4,602 & 4,572 & 4,465 \\
\hline 4,513 & 4,599 & 4,636 & 4,692 & 4,760 & 4,721 & 4,656 & 4,610 & 4,498 \\
\hline 4,559 & 4,634 & 4,670 & 4,741 & 4,803 & 4,771 & 4,702 & 4,660 & 4,541 \\
\hline 4,534 & 4,616 & 4,640 & 4,692 & 4,755 & 4,717 & 4,653 & 4,618 & 4,456 \\
\hline 4,480 & 4,583 & 4,589 & 4,626 & 4,676 & 4,642 & 4,590 & 4,566 & 4,459 \\
\hline 4,463 & 4,505 & 4,568 & 4,631 & 4,667 & 4,650 & 4,616 & 4,539 & 4,488 \\
\hline
\end{tabular}

Estos datos permiten determinar que la celda fotovoltaica captura el haz de luz en los diferentes puntos del área de la máquina con un voltaje adecuado para su acondicionamiento.

Dicho circuito de acondicionamiento del sensor (Figura 4), el cual se conecta a la entrada de una tarjeta Arduino Uno, para así proceder a la toma de datos e iniciar el proceso de diseño del controlador.

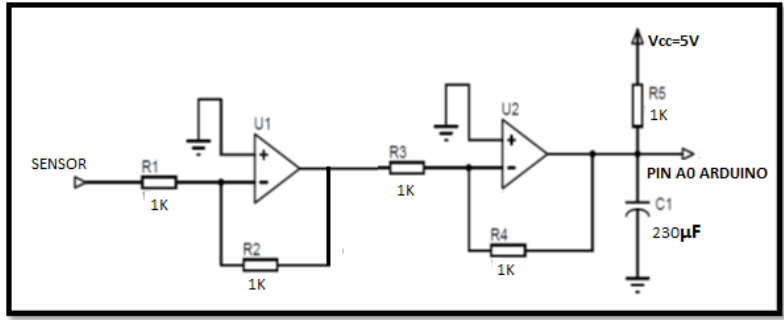

Figura 4. Circuito acondicionador del sensor.

Con el circuito de acondicionamiento mostrado en la figura 4 se obtiene la gráfica en Matlab que representa la variación del sensor con respecto a la entrada generada. La señal de salida comprendida entre un valor de 0 voltios a 5 voltios, con una frecuencia de oscilación de $1 \mathrm{KHz}$. La señal generada en en la adquisición de datos en Matlab inicia con un valor de 0 voltios, con incrementos de 0.05 voltios hasta llegar al valor máximo de 5 voltios, con un tiempo de muestreo en la adquisición de 0.02 segundos.

En la Figura 5 se aprecia la respuesta del sensor graficada en Matlab con el código de adquisición de datos.

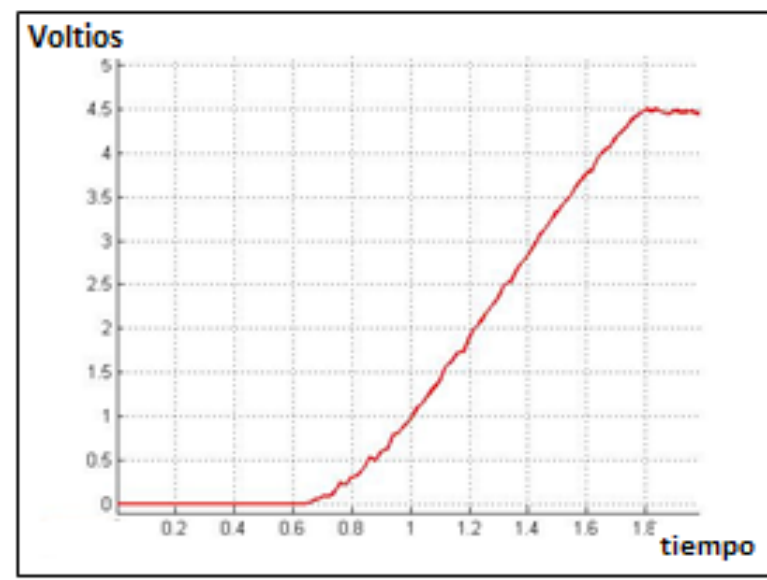

Figura 5. Grafica de la respuesta del sensor

A continuación se ingresan estos datos adquiridos en Matlab con la función Ident, definiendo el intervalo de muestreo y el tiempo de inicio, cmo se observa en la figura 6, de esta manera se procesan los datos, generando la caracterización de la planta. 


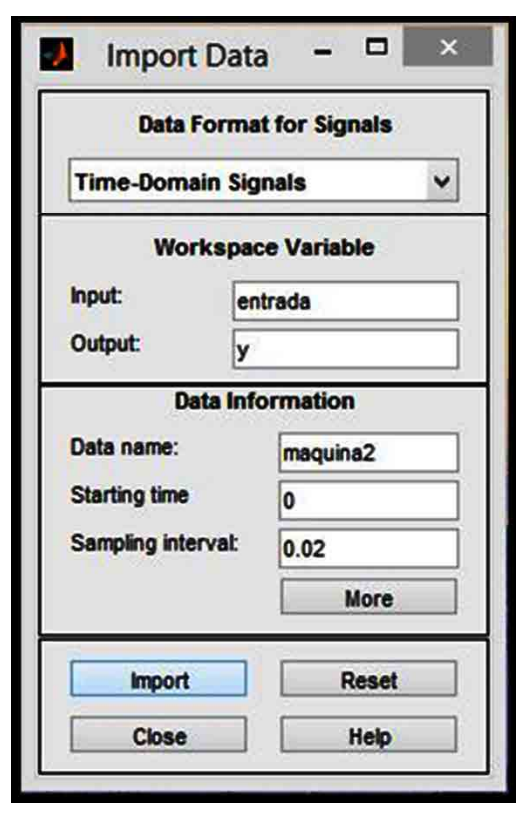

Figura 6. Datos importados del Workspace de Matlab

En la función Ident se aprecia los datos adquiridos para luego ser procesados y generar la ecuación de transferencia de la máquina. Figura 7.

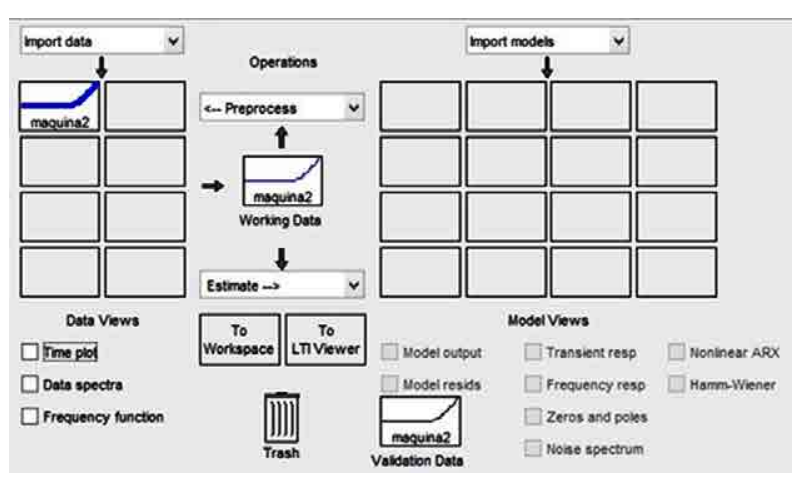

Figura 7. Función Ident de matlab
Se procede a evaluar los datos de entrada. La descripción matemática de un polo real y retardo se obtiene con una aproximación del $90 \%$ utilizando la siguiente definición en la herramienta Ident de Matlab. Ver Figura 8.

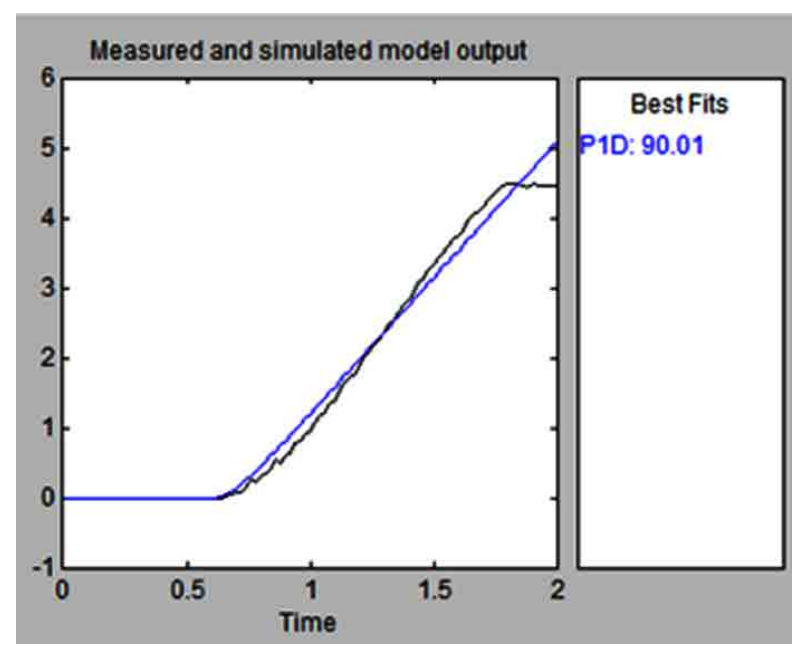

Figura 8. Grafica de estimación P1D.

Estos datos se exportan al workspace de Matlab y se obtiene la función de transferencia de la máquina de insolación, como se aprecia en la Figura 9.

La expresión allí encontrada corresponde a un sistema de primer orden, con retardo de transporte, como lo indica la ecuación (4), cuya constante de tiempo es de 0.6 segundos. A partir de esta se precede a realizar el análisis con el comando pade (aproximación del retardo de transporte) de Matlab para definir la ecuación de la función de transferencia de la máquina en el tiempo continuo. Este resultado se presenta en la figura 10.

$$
P L A N T A=\frac{K e^{-L s}}{T s+1} \text { (4) }
$$




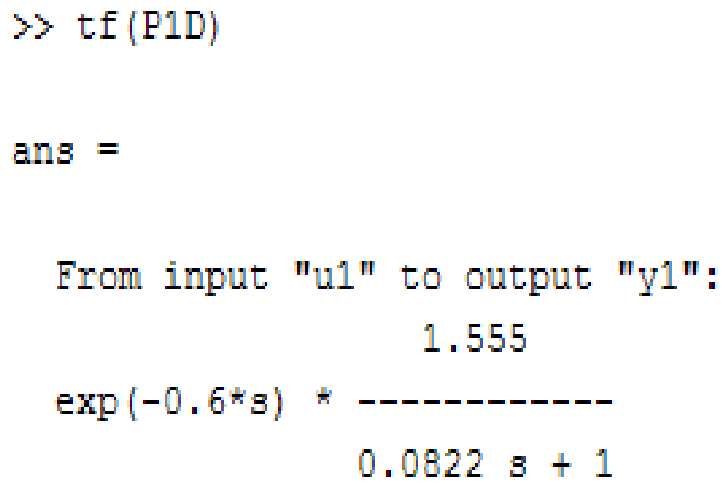

Name: P1D

\section{Continuous-time transfer function.}

Figura 9. Command window de Matlab de la respuesta P1D.

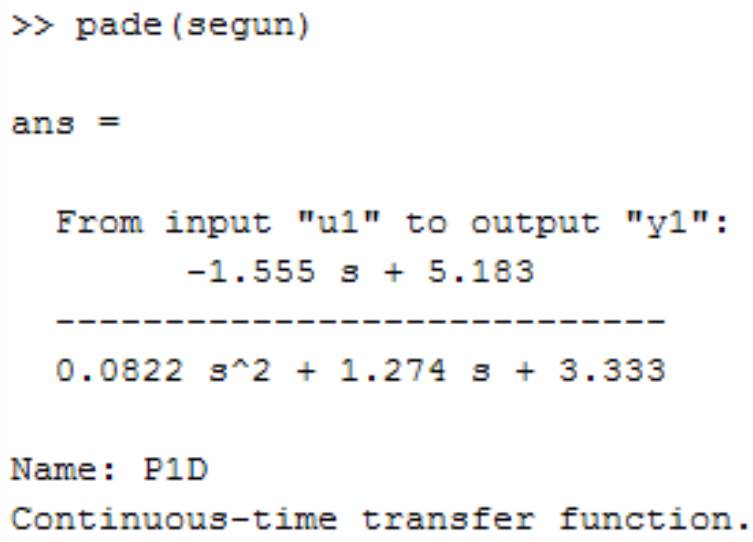

Figura 10. Función de transferencia aproximada con comado pade.

Puede notarse que la función de transferencia allí encontrada corresponde a un sistema que presenta un cero y dos polos. A continuación se evalúa dicha función de transferencia con el comando rlocus, cuyo resultado se observa en la figura 11.

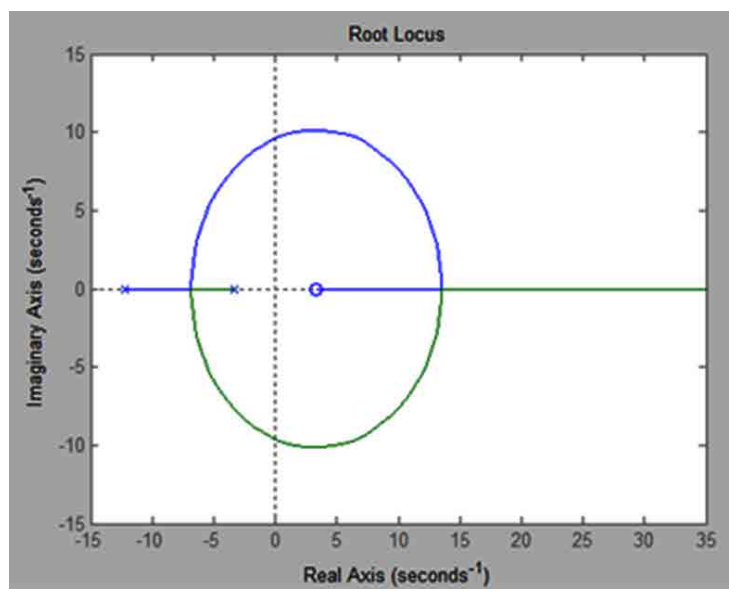

Figura 11. Lugar geométrico de las raíces de P1D.

Como se puede apreciar, la ubicación de los polos que se encuentran en el semiplano negativo del plano indican una respuesta estable, lo cual garantiza que el sistema logrará llevar las señal de salida del sistema al valor deseado, el cual se establecerá en el set point de la máquina de insolación.

Habiendo definido la función de transferencia de la planta, se procede a calcular un controlador con la herramienta PIDtool de Matlab, encontrando las constantes del controlador así:

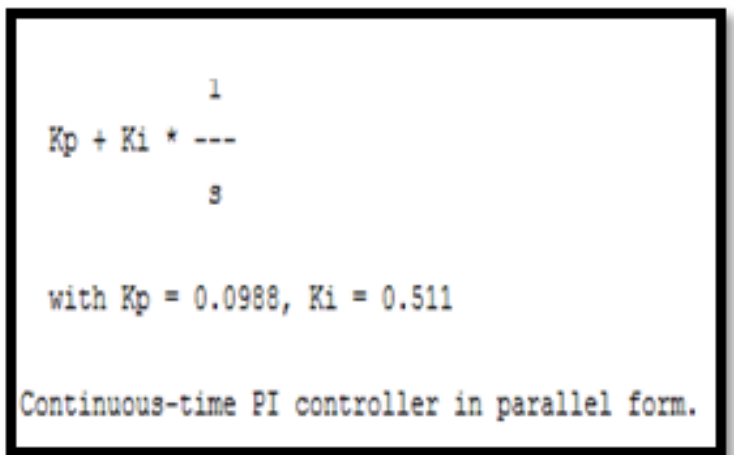

Figura 12. Constantes controlador PI

Con la herramienta Sisotool de Matlab se puede observar el lugar geométrico de las raíces de la ecuación P1D pade con el controlador PI., como se muestra en la figura 13. 


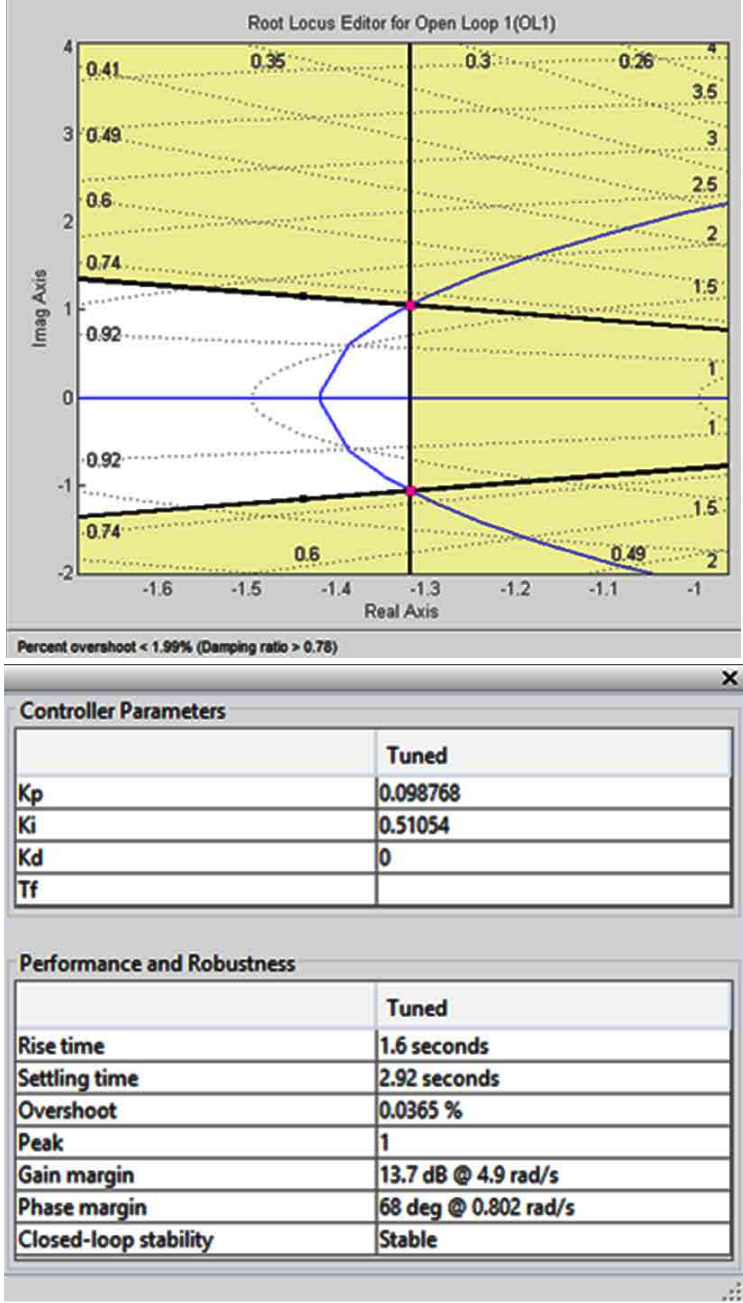

Figura 13. Lugar geométrico de las raíces de P1D. Requerimientos de diseño Sisotool de Matlab.

\section{RESULTADOS}

Hasta el momento se han obtenido resultados satisfactorios en el proceso de diseño, con las herramientas que ofrece Matlab, ahora se presentan los resultados que de ellos derivan, especialmente con respecto a las curvas de respuesta de controlador.

El sistema hallado se evalúa ahora con una señal escalón y luego una señal impulso, las cuales permiten el análisis de la estabilidad del sistema en el dominio del tiempo, siendo esta una de las características más usuales en un diseño de controladores. Las figuras 14 y 15 muestran la respuesta del sistema ante una señal escalón y ante una señal impulso, respectivamente.
La respuesta en el tiempo de un sistema de control consta de dos partes: la respuesta transitoria y la respuesta en estado estacionario. La respuesta transitoria se refiere a la que va del estado inicial al estado final. Por respuesta en estado estacionario se entiende la manera como se comporta la salida del sistema conforme $t$ tiende a infinito. [7].

Por tanto, la respuesta del sistema $\mathrm{c}(\mathrm{t})$ se puede escribir como se muestra en la ecuación 5 .

$$
C(t)=C t r+\operatorname{Css}(t) \quad(5)
$$

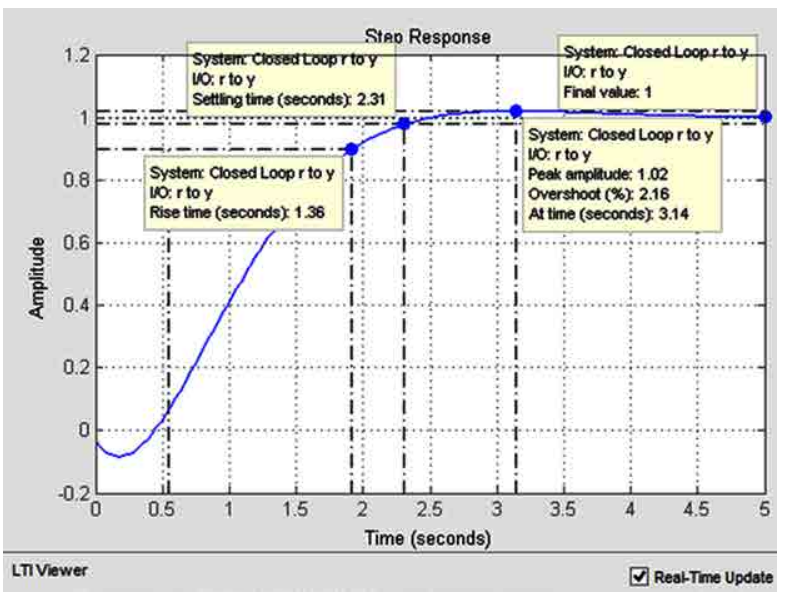

Figura 14. Respuesta escalón del controlador P1D.

Como se puede apreciar, la respuesta en el dominio del tiempo ante una señal escalón muestra la estabilidad del controlador diseñado, en un tiempo bajo, aproximadamente 3 segundos, además el sobrepaso máximo es de tan solo $2.16 \%$,

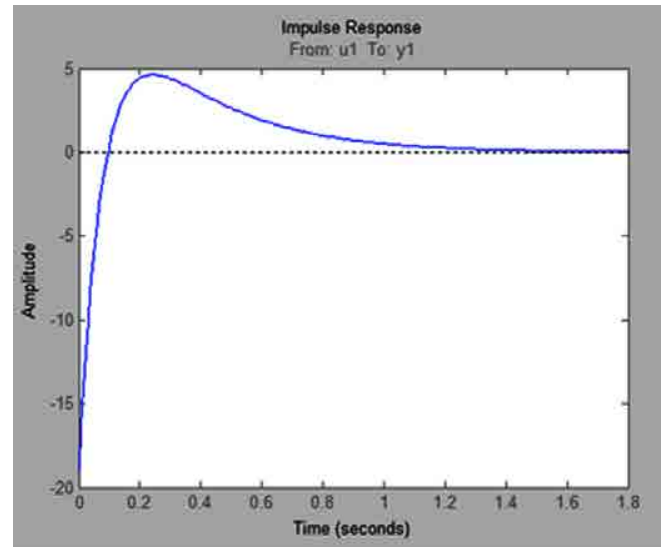

Figura 15. Respuesta impulso del controlador P1D. 
De igual manera la respuesta del sistema ante una función impulso presenta una respuesta rápida, logrando estabilizarse en 1.4 segundos, lo cual permite concluir que los valores encontrados para las constantes del controlador actúan de manera adecuada.

Una vez se ha verificado la estabilidad del sistema, se analiza la ecuación con la herramienta PIDtool de Matlab para encontrar un controlador PID.

Es importante el uso de la herramienta Sisotool de Matlab para verificar mediante el lugar geométrico de las raíces la ecuación P1D pade con el controlador y los resultados del diseño, como se muestra en la figura 15, donde se aprecia un sobrepaso menor al $4 \%$, lo cual representa una respuesta bastante importante para el controlador.

\section{ANÁLISIS DE RESULTADOS}

Los resultados presentados hasta este momento representan un desempeño adecuado para la máquina de insolación; ahora se procede a realizar un análisis de ellos, como lo es en la gran mayoría de los casos la respuesta paso del sistema, pero esta vez los datos obtenidos del controlador y la función de transferencia son llevados a Simulink de Matlab, como se muestra en la figura 16. Allí también se evaluará con una respuesta paso, teniendo así en la simulación una respuesta más real del sistema. La respuesta que se obtiene a la salida del osciloscopio se observa a continuación en la figura 17, en la cual la línea de color amarillo representa la salida del contro- lador y la línea de color morado representa la salida de la planta.

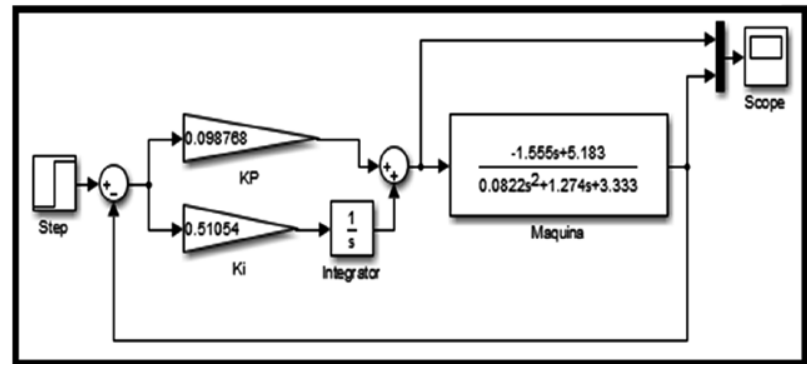

Figura 16. Simulación en Simulink de Matlab

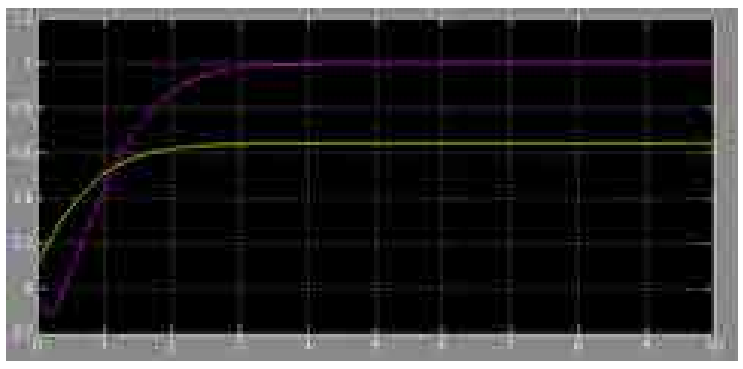

Figura 17. Salida del controlador y la planta

A partir de este se implementan los datos obtenidos del controlador y la función de transferencia de P1D en Simulink de Matlab, llevándolo al diagrama del controlador presentado en la figura 18 para implementarlo en la tarjeta Arduino Uno.

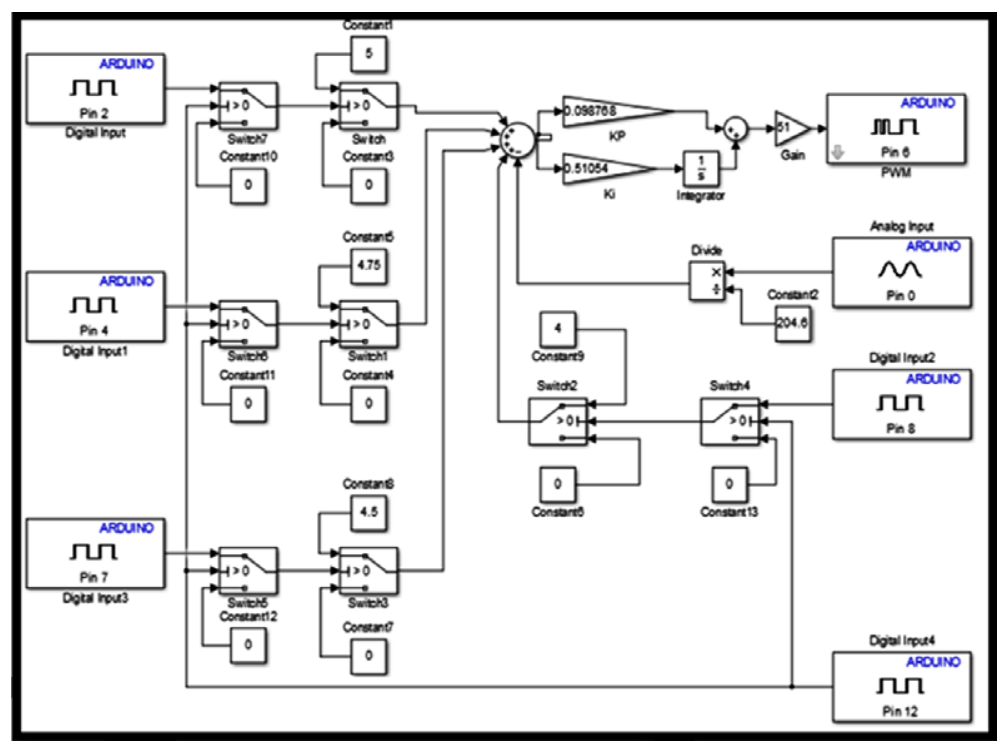

Figura 18. Programa implementado en tarjeta Arduino Uno. 
Puede concluirse dentro del presente análisis que el programa elaborado en Simulink de Matlab mediante el diagrama en bloque es adecuado, la tarjeta Arduino lo reconoce adecuadamente, por lo tanto se procede a generar el código en lenguaje Arduino utilizando la herramienta de PID que incorpora dicho lenguaje. A continuación se describe el programa realizado en Arduino.

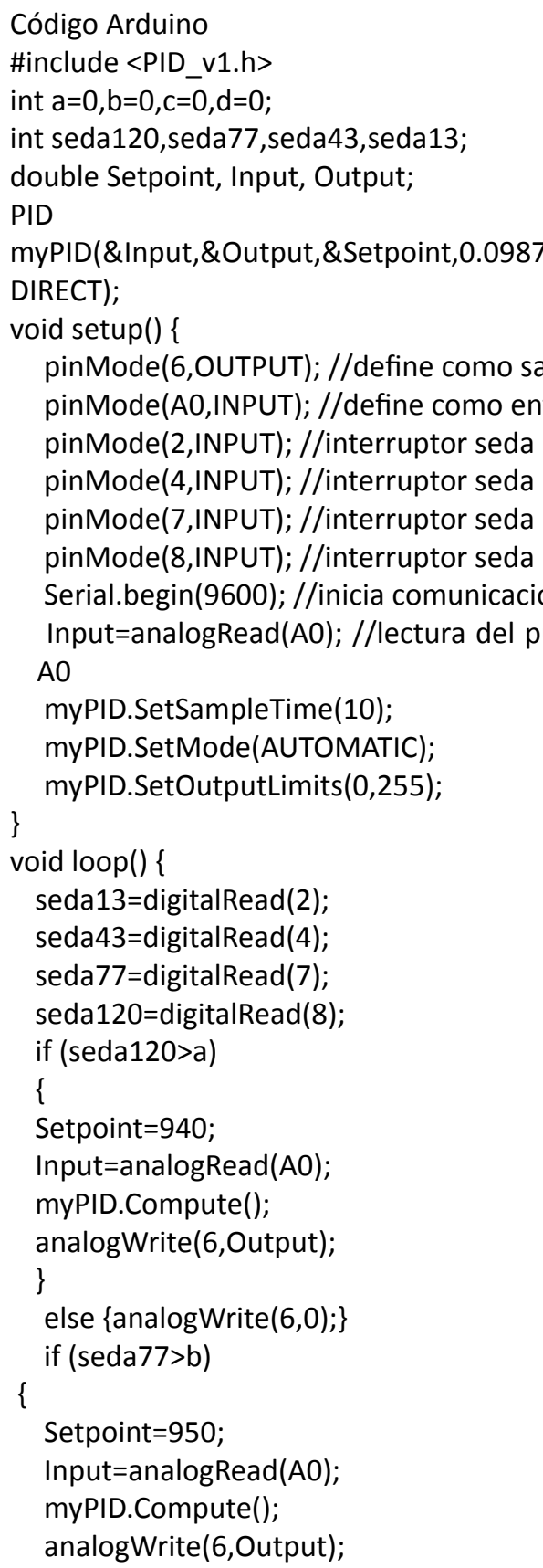

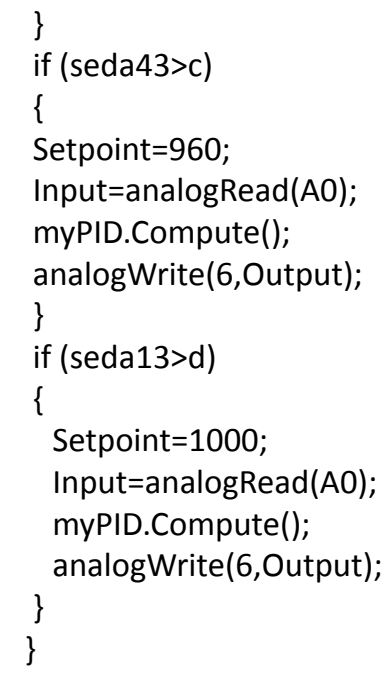

Como punto importante del sistema propuesto se indican las lámparas planteadas para la máquina, las cuales permiten una mayor duración en horas de funcionamiento gracias a la tecnología LED. En la figura 19, se muestra la diferencia en la luz generada por la máquina propuesta (luz LED blanca) y la máquina actual (luz amarilla), siendo factor preponderante la reducción en el consumo de energía para la máquina de insolación propuesta,

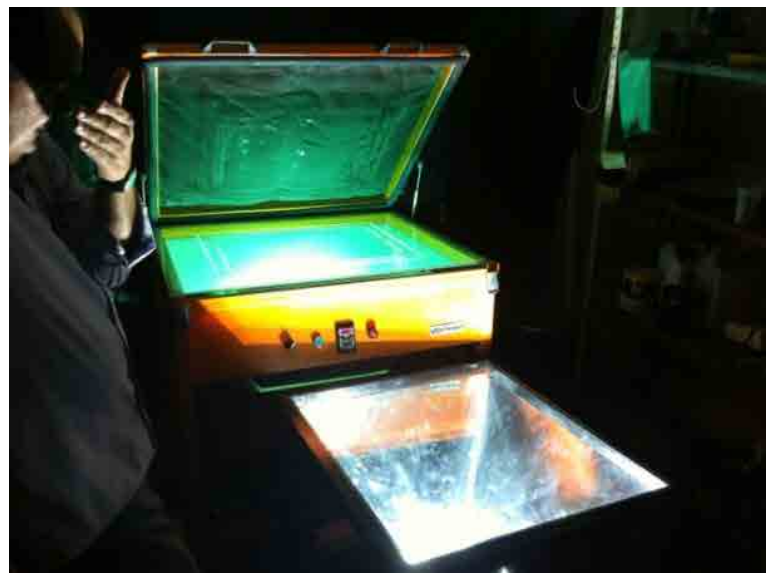

Figura 19. Prototipo máquina de insolación con tecnología LED

En la figura 20 se presenta el resultado del proceso de fotocurado con la máquina de insolación diseñada, en la cual se observa un buen resultado en la definición logrando eliminar los resultados de definición presentados con máquina actual. 


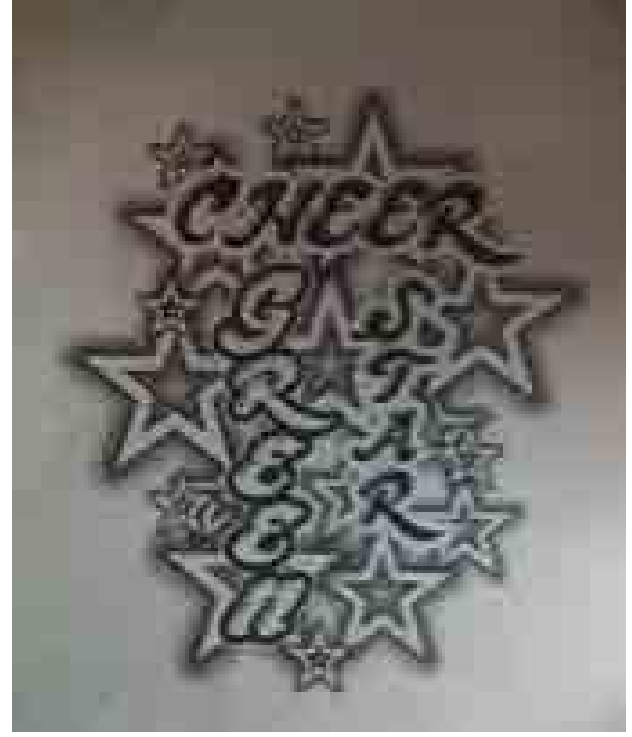

Figura 20. Positivo hecho en prototipo máquina de insolación con tecnología LED

\section{CONCLUSIONES}

La máquina de insolación propuesta con el controlador PI permite establecer un mejor diseño en el proceso de fotocurado eliminando los errores de definición, logrando una imagen clara y de mejor calidad.

Las celdas fotovoltaicas permiten la captación de la intensidad de luz proveniente de las lámparas LED de forma adecuada, entregando una señal de voltaje DC de fácil acondicionamiento.

Con las lámparas LED instaladas se logró un mejor desempeño en la máquina de insolación, permitiendo el proceso de fotocurado en el mismo tiempo, sin importar el tipo de seda, además se redujo el consumo de energía.

La tarjeta Arduino uno presenta un buen funcionamiento en el control del proceso, pero para ajustes bajos de la referencia se requiere un procesamiento mayor.

La máquina de insolación propuesta permite una mayor facilidad en su uso, no requiriendo un personal cualificado para su puesta en funcionamiento.

Los valores de las constantes obtenidas para el controlador permiten establecer un diseño adecuado, ratificado en las respuestas del sistema a partir de las respuestas obtenidas de Matlab.

Se logró una buena caracterización de la planta con un valor del $90 \%$ de la curva de respuesta del sensor siendo este muy cercano a la respuesta original dando como resultado una función de transferencia muy similar a la real.

Si se desea implementar un sistema más robusto de control se aconseja la utilización de una tarjeta de mayor procesamiento para mejorar el tiempo de muestreo y respuesta al actuador.

\section{REFERENCIAS}

[1] C. A. Bernal Torres, "Desarrollo de la Investigación y reporte del informe final,". in Metodología de la Investigación, O. Fernández Palma, Pearson Educación de Colombia Ltda. $3^{\mathrm{a}}$ ed. Colombia, 2010, pp. 234-258.

[2] M. Gonzalez, A. Noguero, F. Pérez, and I. Calvo, "Reconfigurando Aplicaciones de Automatización Industrial Con FTT-MA," in XXXIV Jornadas de Automática, 2013, pp. 767-72.

[3] A. Creus, A. "Medidas de Caudal," in Instrumentación Industrial, Miembro de la Cámara Nacional de la Industria Editorial Mexicana, Alfaomega Grupo Editor, S.A. de C.V. $8^{\text {va }}$ ed. México, 2011, pp. 195220.

[4] R. Piedrafita M., Ingeniería de la Automatización Industrial, Alfaomega, 2da Edición ampliada y actualizada, México, 2010, pp. 59-80.

[5] V. Ruíz, "Métodos de sintonización de controladores PID que operan como reguladores". Revista Ingeniería, vol $12, \mathrm{n}^{\circ} 2$, pp. 21-36, 2011. DOI: https://doi.org/10.15517/ring.v13i1-2.617

[6] A. R. Pallas, Sensores y Acondicionadores de Señal, Marcombo, S.A., $4^{\text {ta }}$ Ed., México: Marcombo, 2005, pp. 40-120.

[7] K. Ogata, "Análisis de la respuesta transitoria y estacionaria" in Ingeniería de Control Moderna. Prentice Hall, $5^{\text {ta }}$ ed., México, 2010, pp. 170-190.

[8] M.A. Pérez García, Instrumentación Electrónica, Garceta grupo editorial, 2012.

[9] G. M. González, "Controlador de lámparas LED con ajuste de la intensidad luminosa," Tesis fin de carrera, Universidad de Cantabria, Jul. 2013. 
[10] R. Hernández G. "Modos de control y diseño de controladores", in Introducción a los Sistemas de Control, Conceptos, Aplicación y simulación con

[11] J. Acedo Sánchez. "Elementos Finales de Control," in Instrumentación y Control Básico de Procesos, Ediciones Díaz de Santos: España, 2006, pp. 353370.

[12] B.C. Kuo,"Diseño de sistemas de Control," in Sistema de Control Automático, Prentice Hall, $7^{\text {ma }}$ ed., México, 2010, pp. 665-714.
[13] A. Morales, S., Instrumentación Básica de Procesos Industriales, International Society of Automation (ISA), México, 2007, pp. 20-40.

[14] The MathWorks. MATLAB Support Package for Arduino Harware User Guide R2014a [online], 2014. Disponible en: http://www.mathworks. com/help/supportpkg/arduinoio/examples/getting-started-with-matlab-support-package-for-arduino-hardware.html 